Revue d'histoire de l'Amérique française

PGS REVUE D.HISTOIRE DE L'AMÉRIQUE FRANÇAISE

\title{
Pour une histoire de l'Institut canadien de Montréal
}

\section{Michelle Guitard}

Volume 27, numéro 3, décembre 1973

URI : https://id.erudit.org/iderudit/303284ar

DOI : https://doi.org/10.7202/303284ar

Aller au sommaire du numéro

Éditeur(s)

Institut d'histoire de l'Amérique française

ISSN

0035-2357 (imprimé)

1492-1383 (numérique)

Découvrir la revue

Citer cette note

Guitard, M. (1973). Pour une histoire de l'Institut canadien de Montréal. Revue d'histoire de l'Amérique française, 27(3), 403-407.

https://doi.org/10.7202/303284ar d'utilisation que vous pouvez consulter en ligne.

https://apropos.erudit.org/fr/usagers/politique-dutilisation/ 


\title{
NOTE DE RECHERCHE
}

\section{POUR UNE HISTOIRE DE L'INSTITUT CANADIEN DE MONTREAL *}

\author{
Michelle GuITARD \\ Hull, Québec
}

Nous connaissons l'importance de l'Institut Canadien de Montréal au siècle dernier, dans le développement du Canada français sur les plans politique, intellectuel et moral. Ses succès autant que ses échecs canalisaient l'opinion et les conflits nationalistes de l'époque. A ce sujet, Jean-Paul Bernard nous a ouvert des avenues intéressantes avec son ouvrage intitulé Les Rouges. La lecture de ce livre prit pour nous une signification particulière. En effet, intrigué par une affirmation de l'auteur selon laquelle le "membership" de l'Institut Canadien de Montréal restait à faire, nous nous sommes résolument mis à la tâche de recueillir les noms de membres dans le but d'établir à travers eux l'histoire sociale de l'Institut.

Les chercheurs ne signalant nulle part l'existence de registres ou de listes des membres de l'Institut, nous avions pris le parti d'établir graduellement cette liste à l'aide de notes prises dans les annuaires, dans les journaux et dans les écrits sur l'époque et sur l'Institut Canadien. Nous n'avons pas tardé à constater que ce mode de collection n'offrait qu'une vue bien partielle du type de personnes appartenant à l'Institut.

L'article de la Constitution de 1845 ne stipulait-il pas que "le secrétaire-archiviste sera le dépositaire des archives de l'Institut; tiendra un journal des procédés de chaque séance et une liste de tous les membres." 1 Il s'ensuit qu'une liste ou des listes devaient exister... La consultation des inventaires d'archives des Rapports des Archives de la Province de Québec et

* Nous remercions M. Jacques Monet, s.j., pour l'aide et l'encouragement reçus afin de continuer cette présente recherche, et Mesdemoiselles Trenholne et Graham, de l'Institut Fraser-Hickson, pour leur coopération très appréciée.

1 Institut Canadien, Constitution et règlements de l'Institut Canadien (Montréal, Impr. Louis Perrault, 1845).

RHAF, vol. 27, no 3 (décembre 1973) 
de plusieurs revues ne nous apprenait rien. Toutefois Lovell C. Clark dans son ouvrage nous apprend que la bibliothèque de l'Institut Canadien a été léguée au Fraser-Hickson Institute de Montréal. ${ }^{2}$ C'est justement là que nous avons découvert de nombreux documents administratifs de l'Institut Canadien dont le très recherché Registre des admissions et résignations, principale source documentaire de notre recherche.

Nous avons alors monté un fichier alphabétique à partir de ce registre. Projet simple au premier abord, mais qui s'avéra plus compliqué quand nous dûmes tenir compte des erreurs que comportait le manuscrit.

A la suite de l'incendie de 1850 , les secrétaires-archivistes ont tenté de rétablir la liste des membres de l'Institut d'avant 1851. Cette dernière liste ne donne que très rarement la date d'admission et de "résignation"; et, on doit aussi se méfier des noms répétés et des réadmissions. Cependant d'après les journaux et autres documents de l'époque, la liste des inscriptions après 1851 paraît avoir été consciencieusement établie à l'exception de quelques erreurs dues aux inscriptions répétées. Ce qui augmente les possibilités d'erreurs, c'est le nombre restreint d'avis de démissions écrites; ainsi s'expliquent probablement plusieurs inscriptions répétées. Les membres qui n'avaient pas payé leur contribution étaient rayés de la liste sans que le registre le signale. Il faudrait probablement vérifier les registres du trésorier.

Une liste exacte des membres de l'Institut exigerait la vérification des prénoms. Ainsi, nous avions trois fiches pour J.-J.-E. Bibaud: une pour J. Bibaud, une autre pour J.-E. Bibaud et une troisième pour E. Bibaud. Les Archives du Canada nous ont révélé qu'il s'agissait de Jean-Jacques-Evariste Bibaud, qu'on dénomme aussi Jean-Baptiste-Evariste. Il y aurait lieu de faire plusieurs vérifications de la sorte. Il arrive que les fiches avec des noms identiques possédant des initiales identiques désignent la même personne; par contre l'identification des nombreux Joseph et Louis ayant les mêmes noms de famille nécessiterait des recherches plus poussées. Quant à l'adhésion, sachons que plusieurs membres ont quitté l'Institut pour y revenir plus tard. Ainsi J.-J.-E. Bibaud, admis le 15 mai 1851, annulait le 24 avril 1862 , était réadmis le 7 octobre 1869 et démissionnait à nouveau le 27 février 1871.

${ }^{2}$ Lovell C. Clark, ed., The Guibord Affair (Holt, Rinehart and Winston of Canada Ltd., Toronto - Montreal, 1971). 
La liste des officiers élus à diverses fonctions de l'Institut peut être recueillie dans les journaux L'Avenir et Le Pays, dans l'Institut-Canadien en 1855, et finalement dans le Mackay's Montreal Directory. Les journaux publient habituellement le résultat des élections à l'Institut dans leurs numéros suivants. - Ce ne fut pas le cas pour les élections de mai 1860. - La liste des officiers que donne l'annuaire de la ville de Montréal dans sa section "Miscellaneous Directory" ne tient compte que du résultat des élections de mai et ne mentionne pas celui des élections de novembre. Treize postes devaient être comblés lors des élections. Mais le comité de la Régie qui comptait quatre membres élus n'exista que de novembre 1845 à novembre 1848. Ainsi à part les sept premières élections, il restait neuf postes à combler aux élections subséquentes: président, premier-viceprésident, second-vice-président, secrétaire-archiviste, assistantsecrétaire-archiviste, secrétaire-correspondant, trésorier, bibliothécaire, assistant-bibliothécaire. Souvent des changements dus à des démissions ou à des absences eurent lieu entre les élections officielles, nécessitant de nouvelles élections pour un poste ou pour plusieurs, telle la séance élective du 8 juin 1848. Le journal des comptes rendus de chaque séance tenu par le secrétairearchiviste nous renseignerait probablement à ce sujet.

L'étude sociale de l'Institut Canadien nécessiterait la connaissance de l'occupation et des activités de chacun des membres. Nous avons constaté l'ampleur du travail entrepris après avoir cherché systématiquement à identifier les membres dont le patronyme commençait par les lettres $\mathrm{A}, \mathrm{B}, \mathrm{C}$, et $\mathrm{D}$, ceci à l'aide des annuaires de la ville de Montréal et de toutes informations cueillies lors de nos lectures. Nous avons dû en rester là, mais ce début de recherche sur l'occupation et les activités des membres de l'Institut nous avait déjà beaucoup renseigné. Les membres des professions libérales, notamment les avocats, sont pour la plupart étudiants lors de leur adhérance à l'Institut. Presque tous en sortent au terme de leurs études. Nous retrouvons, outre les avocats, les médecins, les notaires, les juges et les étudiants, des professeurs, des artistes ainsi qu'un grand nombre de commis, de commis-marchands, et de marchands. La classe ouvrière y est fortement représentée.

Le recours au Mackay's Montreal Directory nous a permis de constater qu'un nombre prépondérant de membres avaient leur adresse d'affaire ou leur adresse résidentielle aux environs de l'Institut Canadien situé alors au 111 Notre-Dame entre les 
rues Gosford et Bonsecours. ${ }^{3}$ Ceci laisse supposer que l'Institut était un lieu de rendez-vous. Ces annuaires montréalais mentionnent presque toujours l'occupation à la suite du nom. On y publie dans une section intitulée "Miscellaneous Directory" le nom des diverses organisations, celui des officiers, de certains membres et des personnes affiliées à ces organisations. Ainsi nous y trouvons le nom des professeurs à l'Ecole de médecine et le titre des cours dont ils sont chargés, ou encore les diverses églises et les prêtres qui y sont affectés, les clubs et leurs dirigeants. On y publie de multiples renseignements sur les postes et officiers de la fonction publique. A partir de 1863-64, une liste des membres du Barreau est donnée avec la date de leur admission au Barreau; cette liste souffre de lacunes, omettant par exemple les noms des avocats travaillant sous l'égide d'une compagnie. Il serait profitable de vérifier à l'aide de cette section 'miscellaneous' les autres tâches cumulées dans les diverses organisations par les membres de l'Institut Canadien, ce qui pourrait nous renseigner sur son rayonnement social. Certains membres surgissent de partout! L'utilisation de ces annuaires confirme la difficulté d'identification de certaines personnes longtemps à l'emploi de leur père ou d'une entreprise familiale. Quand ces jeunes membres habitent sous le toit familial, il faut attendre plusieurs années pour les identifier, attendre qu'ils aient pris résidence séparée ou qu'ils aient pris charge du commerce paternel. Leur occupation est alors manifeste grâce aux titres de ce genre: 'of John Smith and Sons' ou 'of such and such Company' ou encore 'of the office of Smith \& Brown'.

Nous avons constaté l'existence plausible de 1,885 membres ayant passé par l'Institut Canadien de 1844 à 1873. La liste des membres réfractaires lors de la scission de 1858 est disponible en vertu de leur démission collective qui a permis l'inscription au registre de la date de démission, le 22 avril 1858. Les départs sont numérotés (ex.: les démissionnaires de 1858 ont tous le numéro 595) permettant ainsi d'établir une courbe statistique. Une pareille courbe reste également à tracer pour les admissions. Nous croyons que la confrontation comparative de ces deux courbes serait, à la lumière des grands événements de l'Institut Canadien, particulièrement intéressante, événements tels l'expulsion de 250 membres en $1847^{4}$, l'incendie de 1850 , la scission

3 Archives de la ville de Montréal, Cadastre no 130. Aujourd'hui ce site est un terrain de stationnement municipal.

4 J.-R. Rioux, "L'Institut Canadien, les débuts de l'Institut Canadien et du journal L'Avenir (1844-1849)". Thèse manuscrite (Université Laval, 1967), 32. 
de 1858, ou encore la condamnation de l'Institut par la Congrégation romaine en $1869^{5}$. Nous avons cru remarquer que les démissions sont presque toujours compensées par une foison d'adhésions. Aussi avons-nous constaté que le nombre d'adhérents anglophones augmentait au rythme des fustigations cléricales lancées contre l'Institut. Ainsi la nouvelle souscription organisée par Joseph Doutre en 1868 attire plusieurs membres anglais mais seulement quelques nouveaux francophones contestataires.

L'Institut Canadien exerçait une attraction considérable: des gens de toutes classes sociales, des personnes de grande renommée du monde des lettres et des sciences, sans mentionner les politiciens, les légistes et les hommes d'affaires. Nous y avons découvert deux noms qui ne sont rien de moins que des sommités de la culture française: Victor Hugo et Michelet, tous deux membres correspondants de l'Institut Canadien à compter de 1870.

Cette ébauche de recherche nous a appris la possibilité d'une étude sociale de l'Institut Canadien de Montréal à partir de ses documents administratifs et du Mackay's Montreal Directory. D'autres documents, tels les registres paroissiaux et les rôles d'évaluation de la ville de Montréal enrichiraient notre recherche d'informations pertinentes aux membres de l'Institut. Il nous faudra éventuellement tenter une évaluation de l'Institut sous son aspect d'organisme social ainsi que de ses membres vus dans leur contexte socio-économique du milieu du XIXe siècle. La supputation de leurs activités, de leurs tâches et de leur contexte social saurait nous jeter de nouvelles lumières sur l'idéologie des "Rouges", et partant sur l'idéologie d'un segment de la population montréalaise de l'époque.

5 J.-P. Bernard, Les Rouges, Libéralisme, nationalisme et anticléricalisme au milieu du XIXe siècle (Montréal, Les Presses de l'Université du Québec, 1971), 317. 